\title{
PRR11 and SKA2 promote the proliferation, migration and invasion of esophageal carcinoma cells
}

\author{
JIE CHEN ${ }^{1}$, HONG-MEI YANG ${ }^{1}$, HUI-CHONG ZHOU ${ }^{2}$, RUI-RUI PENG $^{1}$, \\ ZHAO-XIAO NIU ${ }^{1}$ and CHUN-YAN KANG ${ }^{1}$ \\ ${ }^{1}$ Department of Pathophysiology, Henan Medical College, Zhengzhou, Henan 450000; ${ }^{2}$ Department of Gastroenterology, \\ Second Affiliated Hospital of Zhengzhou University, Zhengzhou, Henan 450000, P.R. China
}

Received April 23, 2019; Accepted April 3, 2020

DOI: $10.3892 / \mathrm{ol} .2020 .11615$

\begin{abstract}
Proline-rich protein 11 (PRR11) together with its upstream adjacent gene, spindle and kinetochore associated 2 (SKA2), represent a classic, head-to-head gene pair. The role of the PRR11 and SKA2 gene pair has been described in various types of cancer, including breast cancer, non-small cell lung cancer, hepatocellular carcinoma and ovarian carcinoma. However, its role in esophageal carcinoma (ESCC) remains unclear. The mRNA expression levels of PRR11 and SKA2 were examined in ESCC surgical specimens. In addition, the role of PRR11 and SKA2 in the proliferation and migratory and invasive capacities of EC9706 and EC109 cell lines was examined. The results from the present study demonstrated that PRR11 and SKA2 expression levels were upregulated in ESCC tissues compared with adjacent normal tissues. Furthermore, PRR11 and SKA2 knockdown significantly inhibited the proliferation and migratory and invasive capacities of ESCC cells. Conversely, PRR11 and SKA2 overexpression significantly promoted the proliferation and migratory and invasive capacities of ESCC cell lines via activation of the AKT signaling pathway and certain markers of epithelial-mesenchymal transition, including Snail and N-cadherin. The results from the present study suggested that the PRR11 and SKA2 gene pair may represent a potential target in the diagnosis and treatment of ESCC.
\end{abstract}

\section{Introduction}

Esophageal squamous cell carcinoma (ESCC), which is one of the most common types of cancer, was the sixth leading cause of cancer-associated mortality in China in 2016 (1). In 2012, an estimated 455,800 new esophageal cancer cases

Correspondence to: Dr Chun-Yan Kang, Department of Pathophysiology, Henan Medical College, 120 Huanghe Road, Zhengzhou, Henan 450000, P.R. China

E-mail: liuruixuankk@163.com

Key words: PRR11, SKA2, esophageal carcinoma, progression and 400,200 deaths were reported (2). Although surgery and radiation therapy are effective therapeutic strategies for certain tumors diagnosed at an early stage, a number of patients eventually progress to advanced stages of cancer (3). Based on previous epidemiologic studies, it is widely accepted that genetics serve a crucial role in the development and progression of ESCC $(4,5)$; however, the underlying mechanisms remain unclear. It is therefore important to fully understand the development and progression of ESCC, in order to identify novel therapeutic targets.

The proline-rich protein 11 (PRR11) gene is located on chromosome 17q22 and contains a bivalent nuclear localization signal, two proline-rich regions and a zinc finger domains (6). In recent years, PRR11 has been reported to serve as a candidate oncogene in various types of cancer, including pancreatic cancer, breast cancer, non-small cell lung cancer, hepatocellular carcinoma and ovarian carcinoma (7-9). PRR11 and its upstream adjacent gene, spindle and kinetochore associated 2 (SKA2), are a classic head-to-head gene pair, driven by a prototypical and bidirectional promoter (10). SKA2, which is involved in the formation of the Ska complex, is involved in the maintenance of the mitotic mid-plateau and shut-down of the spindle checkpoint (11-14). Currently, studies on PRR11 and SKA2 mainly focus on lung and breast cancer $(7,8,10)$; however, the role of PRR11 and SKA2 in ESCC remains unclear.

The present study evaluated the importance of PRR11 and SKA2 in the progression of ESCC, and demonstrated that the expression level of these genes was highly expressed in human ESCC tissues. Furthermore, the present study revealed that PRR11 and SKA2 serve important roles in the proliferation and migratory and invasive capacities of ESCC cells in vitro. In addition, the underlying mechanism of PRR11 and SKA2 in the progression of ESCC was investigated.

\section{Materials and methods}

ESCC tissue samples. A total of 30 pairs of ESCC and adjacent normal tissues were obtained from patients following resection surgery at the Second Affiliated Hospital of Zhengzhou University between January 2014 and December 2017. The adjacent normal tissue $<1-\mathrm{cm}$ away from the tumor tissue. The median age was 52 (41-61) years old, and 
there were 22 males and 8 females. Some clinical parameters, such as sex, age, history of drinking and smoking, tumor site, TNM staging (the 8th edition) (15), and tumor differentiation. All patients provided informed signed consent. The specimens were immediately stored at $-80^{\circ} \mathrm{C}$ until RNA extraction. The present study was approved by the Ethics Committee of the Second Affiliated Hospital of Zhengzhou University.

RNA preparation and reverse transcription-quantitative polymerase chain reaction analysis. Total RNA was extracted from the 30 paired ESCC tumor tissues and adjacent normal tissues using TRIzol ${ }^{\circledR}$ reagent (Invitrogen; Thermo Fisher Scientific, Inc.) according to the manufacturer's instructions, and $2 \mu \mathrm{g}$ RNA was reverse transcribed to cDNA using the Reverse Transcription System (A3500, Promega Corporation) according to the manufacturers' instructions. The mRNA level was detected using a PikoReal 96 Real-Time PCR system (Thermo Fisher Scientific, Inc.) and SYBR ${ }^{\circledR}$ Premix Ex Taq (Takara Bio, Inc.). The thermal cycling conditions were as follows: 40 cycles at $95^{\circ} \mathrm{C}$ for $20 \mathrm{sec}, 60^{\circ} \mathrm{C}$ for $30 \mathrm{sec}$, and $72^{\circ} \mathrm{C}$ for $30 \mathrm{sec}$, and one cycle of $72^{\circ} \mathrm{C}$ for $10 \mathrm{~min}$. The sequences of the primers were as follows: PRR11, forward 5'-GAAGCT GGCTAACATCATCCTG-3', reverse 5'-CTCTGGGTTATG CAGTTCTGG-3'; SKA2, forward 5'-GGAACTGATGTT CCAGAAAGCTG-3', reverse 5'-AGCTCCAGGTCTGTT TGCTT-3'; and GAPDH, forward 5'-ATGACCCCTTCATTG ACCTCA-3' and reverse 5'-GAGATGATGACCCTTTTG GCT-3'. The relative mRNA expression level in each sample was calculated using the comparative expression level $2^{-\Delta \Delta C q}$ method (16).

Cell culture. The esophageal squamous cell carcinoma cell lines EC9706 and EC109, and 293T cell line were purchased from The Cell Bank of Type Culture Collection of the Chinese Academy of Sciences. Cells were cultured in Dulbecco's Modified Eagle Medium (DMEM) supplemented with $100 \mathrm{U} / \mathrm{ml}$ penicillin, $100 \mathrm{mg} / \mathrm{ml}$ streptomycin, and $10 \%$ (v/v) fetal bovine serum (FBS; Invitrogen; Thermo Fisher Scientific, Inc.) at $37^{\circ} \mathrm{C}$ in a humidified atmosphere containing $5 \% \mathrm{CO}_{2}$.

Plasmids and cell transfection. A PRR11 and SKA2 coding sequence was constructed and inserted into a pcDNA3.1-Myc plasmid (Invitrogen; Thermo Fisher Scientific, Inc.) to generate the PRR11 and SKA2 overexpression vector. Lentiviral supernatants were produced using the Lenti-X HTX packaging system (Clontech Laboratories, Inc.) according to the manufacturer's protocol and used for transfection of EC109 cells. For negative controls, EC109 cell were transfected with supernatants from empty vectors. A total of $30 \mu 1$ lentiviral particles $\left(5 \times 10^{7} \mathrm{UT} / \mathrm{ml}\right)$ were suspended in DMEM, and incubated with the EC109 cells $\left(1 \times 10^{6}\right)$ in a 6-well plate for $12 \mathrm{~h}$ until they reached $70 \%$ confluence. Next, transfected cells were screened by green fluorescence using flow cytometry (IX-71; Olympus Corporation). Lentiviral short hairpin (sh)RNA targeting PRR11 and SKA2 were designed using software provided by Qiagen, Inc. and inserted into a pLKO.1-TRC vector (Han Heng Biotechnology Co., Ltd.). The lentivirus was packaged in 293T cells and collected following centrifugation at $4^{\circ} \mathrm{C}$ and $1,000 \mathrm{x}$ g for $2 \mathrm{~h}$. A total of $50 \mu \mathrm{l}$ lentiviral particles $\left(1 \times 10^{8} \mathrm{UT} / \mathrm{ml}\right)$ were suspended in DMEM, and incubated with the EC9706 cells $\left(1 \times 10^{6}\right)$ in a 6 -well plate for $8 \mathrm{~h}$ until they reached $60 \%$ confluence. Positive EC9706 cells were selected by puromycin $(4 \mu \mathrm{g} / \mathrm{ml})$ (Han Heng Biotechnology Co., Ltd.) for at least 3 days or sorted by flow cytometry. Subsequent experiments were conducted at $48 \mathrm{~h}$ post-transfection. The expression of PRR11 and SKA2 in the resistant clones was further confirmed by western blotting. The sequences of the shRNAs were as follows: shPRR11, forward: 5'-CCGGCCAGAGTTTAGAAG TATTGAACTCGAGTTCAATACTTCTAAACTCTGGTT TTTG-3' and reverse: 5'-AATTCAAAAACCAGAGTTTAG AAGTATTGAACTCGAGTTCAATACTTCTAAACTCTG G-3'; shSKA2, forward: 5'-CCGGCAAACTTTGTATGC CCGCTTTCTCGAGAAAGCGGGCATACAAAGTTGTTT TTG-3' and revere 5'-AATTCAAAAACAAACTTTGTA TGCCCGCTTTCTCGAGAAAGCGGGCATACAAAGTTT G-3'; and shcontrol, forward: 5'-TGACAAGTGGAACCA GAT-3' and reverse: 5'-TGTCCCCACTCACGAAGG-3'.

Western blotting. EC9706 and EC109 cells were lysed in RIPA lysis buffer (Thermo Fisher Scientific, Inc.) for at least $30 \mathrm{~min}$ on ice. The lysates were centrifuged at 10,000 $\mathrm{x}$ g for $20 \mathrm{~min}$ at $4^{\circ} \mathrm{C}$. Protein concentration was determined using Bradford reagent (Sigma-Aldrich; Merck $\mathrm{KGaA}$ ) according to the manufacturer's instructions. Proteins (10 $\mu \mathrm{g} / \mathrm{lane})$ were separated by $10 \%$ SDS-PAGE and transferred onto polyvinylidene fluoride membranes. Then, membranes were blocked with $5 \%$ fat-free milk for $1 \mathrm{~h}$ at room temperature. Next, membranes were incubated with primary antibodies at $4^{\circ} \mathrm{C}$ overnight. The primary antibodies were as follows: PRR11 (1:500; cat. no. 3220; Cell Signaling Technology, Inc.), SKA2 (1:500; cat. no. 2419; Cell Signaling Technology, Inc.), phosphorylated (p) and total Akt (1:1,000; cat. no. 10176-2-AP; ProteinTech Group, Inc.), proliferating cell nuclear antigen (PCNA; 1:1,000; cat. no. 10205-2-AP; ProteinTech Group, Inc.), Snail (1:1,000; cat. no. 13099-1-AP; ProteinTech Group, Inc.), Cyclin D1 (1:1,000; cat. no. 60186-1-Ig; ProteinTech Group, Inc.), N-cadherin (1:1,000; cat. no. 22018-1-AP; ProteinTech Group, Inc.) and GAPDH (1:1,000; cat. no. 60004-1-Ig; ProteinTech Group, Inc.). Snail and N-cadherin are markers of EMT, and cyclin D1 is a marker of the cell cycle $(17,18)$. Membranes were then incubated with anti-rabbit horseradish peroxidase-conjugated secondary antibody (cat. no. 7074; 1:2,000; Sangon Biotech Co., Ltd.) for $2 \mathrm{~h}$ at room temperature. The immunoreactive protein bands were visualized using an enhanced chemiluminescence kit (Pierce; Thermo Fisher Scientific, Inc.) and a Gel Dox XR system (Bio-Rad Laboratories, Inc.).

Crystal violet assay. Control and transfected cells were seeded into 6-well plates at a density of 1,000 cells/well. Cells were cultured in DMEM with 10\% FBS and medium was changed every three days. After two weeks of culture, the culture medium was removed and cells were stained with crystal violet ( $1 \mathrm{ml} 0.5 \%$ crystal violet solution in 20\% methanol) for $10 \mathrm{~min}$ at room temperature. Cells were washed with PBS and images were captured using a digital camera. The optical density (OD) value was measured at $600 \mathrm{~nm}$ with a microplate reader. 
Cell proliferation assay. EC9706 and EC109 cells were seeded in 96-well plates at a density of 1,000 per well in triplicate. MTT solution (20 $\mu \mathrm{l} ; 5 \mathrm{mg} / \mathrm{ml})$ (APExBIO Technology LLC) was added to the medium and incubated at $37^{\circ} \mathrm{C}$ for $4 \mathrm{~h}$. The medium was removed, and $200 \mu \mathrm{l}$ DMSO was added to dissolve the formazan crystals. The absorbance was measured using an automatic microplate reader at a wavelength of $490 \mathrm{~nm}$.

Boyden chamber assay. A total of $2 \times 10^{5}$ EC9706 and EC109 cells were loaded into the upper chamber of a 10-well Boyden Chamber (Neuro Probe, Gaithersburg, MD, USA) with a polycarbonate membrane of $8 \mu \mathrm{m}$ pore sizein $200 \mu \mathrm{l}$ of medium containing $1 \%$ FBS. Medium (250 $\mu \mathrm{l})$ containing $10 \%$ FBS was added to the bottom well of the chamber. Cells were incubated for at $37^{\circ} \mathrm{C}$ for $6 \mathrm{~h}$. Cells that have migrated through the pores into the lower chamber were stained with hematoxylin and eosin for $10 \mathrm{~min}$ at room temperature. Cells were then then photographed using an inverted microscope (OLYMPUS) at magnification, x400. Cells were counted from four randomly selected fields by ImageJ software (version 1.8.0, National Institutes of Health). Experiments were repeated in triplicate.

Transwell assay. ESCC cell invasion was examined using a Transwell assay with polyethylene terephthalate membranes (24-well inserts; $8.0 \mu \mathrm{m}$; Corning Inc.). Cell suspension $(150 \mu \mathrm{l})$ containing $2 \times 10^{5}$ cells was loaded into the upper well that was precoated with $20 \%$ Matrigel (BD Biosciences) at $37^{\circ} \mathrm{C}$ for $3 \mathrm{~h}$. DMEM $(500 \mu \mathrm{l})$ containing $10 \% \mathrm{FBS}$ was added to the bottom of the well. After $48 \mathrm{~h}$, cells that have invaded the bottom of the membrane were stained with $0.1 \%$ crystal violet for $10 \mathrm{~min}$ at room temperature and then photographed using an inverted light microscope (Olympus Corporation) at 400x magnification Cells were counted from four randomly selected fields by ImageJ software (version 1.8.0, National Institutes of Health). Experiments were conducted in three independent experiments.

Statistical analysis. Data are presented as the mean \pm standard deviation and statistical analyses were performed using GraphPad Prism software (version 5; GraphPad Software, Inc.). PRR11 and SKA2 mRNA levels between tumor and adjacent normal tissue groups were analyzed using the paired Student's t-test. PRR11 and SKA2 mRNA levels in the cell lines were determined using an unpaired Student's t-test. Comparisons of multiple groups were analyzed using the ANOVA followed by Tukey's post hoc test. $\mathrm{P}<0.05$ was considered to indicate a statistically significant difference.

\section{Results}

PRR11 and SKA2 are upregulated in ESCC tissues. The primary focus of the present study was the expression level of PRR11 and SKA2 in the 30 pairs of ESCC and adjacent normal tissues. The clinicopathological characteristics of the 30 patients are presented in Table I. The results demonstrated that PRR11 expression level was significantly increased in ESCC tissues compared with the adjacent normal tissues $(\mathrm{P}<0.001$; Fig. 1A). Similarly, SKA2 expression level was significantly increased in tumor tissues compared with normal
Table I. Clinicopathological characteristics of the 30 patients included in the present study.

\begin{tabular}{lr}
\hline Variables & Number $(\%)$ \\
\hline Sex & \\
Male & $23(76.7)$ \\
Female & $7(23.3)$ \\
Age, years & \\
$<60$ & $19(63.3)$ \\
$\geq 60$ & $11(36.7)$ \\
Smoking & \\
Yes & $17(56.7)$ \\
No & $13(43.3)$ \\
Drinking & \\
Yes & $12(40.0)$ \\
No & $18(60.0)$ \\
Tumor site & \\
Cervical & $2(6.7)$ \\
Upper & $9(30.0)$ \\
Middle & $10(33.3)$ \\
Lower & $9(30.0)$ \\
TNM stage & $7(23.3)$ \\
IA & \\
IB & \\
IIA & \\
IIB & \\
IIIA & $5(16.7)$ \\
IIIB & $9(30.0)$ \\
Differentiation & $7(23.3)$ \\
Well & $5(16.7)$ \\
Moderate & $2(6.7)$ \\
Poor & $2(6.7)$ \\
\hline
\end{tabular}

TNM stage (15), Tumor-Node-Metastasis.

tissues $(\mathrm{P}<0.05$; Fig. 1B). Furthermore, the mRNA and protein levels of PRR11 and SKA2 in the two ESCC cell lines EC109 and EC9706 were assessed. The results demonstrated that the mRNA level of both PRR11 and SKA2 were significantly higher in EC9706 cells compared with EC109 cells. The protein expression of both PRR11 and SKA2 was also higher in EC9706 cells (Fig. 1C and D).

PRR11 and/or SKA2 knockdown inhibits the proliferation, migration, and invasion of ESCC. To identify the function of the PRR11 and SKA2 gene pair in ESCC cells, specific shRNA targeting PRR11 and/or SKA2 were used in EC9706 cells. The efficiency of these knockdowns was confirmed by western blotting (Fig. 2A). Furthermore, the results from MTT and crystal assays demonstrated that transfection with ShPRR11 or SKA2 significantly reduced EC9706 cell proliferation compared with untransfected cells. In addition, double transfection induced a more significant decrease in cell proliferation compared with single transfections $(\mathrm{P}<0.01$ 

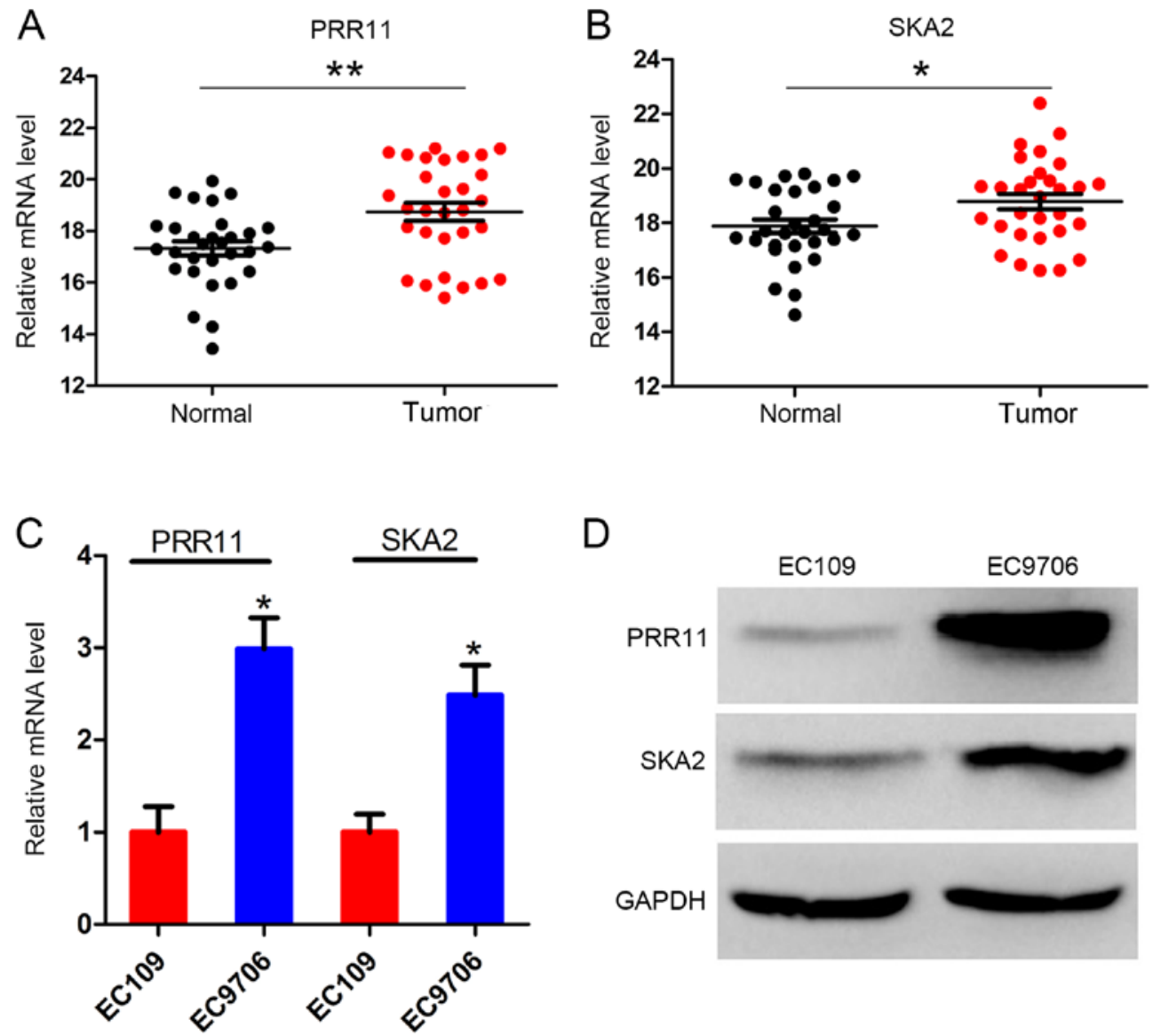

Figure 1. The expression of PRR11 and SKA2 in ESCC tissues and cell lines. (A) PRR11 mRNA levels in 30 pairs of tumor samples and matched normal tissues determined by RT-qPCR. (B) SKA2 mRNA levels in 30 pairs of tumor samples and matched normal tissues determined by RT-qPCR. (C) PRR11 and SKA2 mRNA expression in the two ESCC cell lines EC109 and EC9706. (D) Protein expression of PRR11 and SKA2 in the two ESCC cell lines EC109 and EC9706 by western blotting. GAPDH was used as a loading control. ${ }^{*} \mathrm{P}<0.05$ and ${ }^{* *} \mathrm{P}<0.01$. PRR11, proline-rich protein 11 ; RT-qPCR, real-time quantitative PCR; SKA2, spindle and kinetochore associated 2.

and $\mathrm{P}<0.001$; Fig. $2 \mathrm{~B}$ and $\mathrm{C}$ ). In addition, as presented in Fig. 2D, the results from Boyden chamber assay demonstrated that the migratory ability of EC9706 cells was significantly decreased following PRR11 or SKA2 knockdown compared with untransfected cells $(\mathrm{P}<0.01$ and $\mathrm{P}<0.001$, respectively; Fig. 2D). Cell migratory capacity was also significantly more decreased following double knockdown of PRR11 and SKA2 compared with single transfections $(\mathrm{P}<0.001$; Fig. $2 \mathrm{C})$. Furthermore, the results from transwell assay indicated that the invasive capacity of EC9706 cells was significantly reduced following PRR11 and/or SKA2 knockdown $(\mathrm{P}<0.01$ and $\mathrm{P}<0.001$; Fig. 2E).

PRR11 and/or SKA2 overexpression promotes the proliferation, migration, and invasion of ESCC. PRR11 and/or SKA2 were overexpressed in EC109 cells by transfection. The successful overexpression of PRR11 and/or SKA2 was confirmed by western blotting (Fig. 3A). The results from MTT and crystal violet assays demonstrated a significantly increased cell proliferation following PRR11 or SKA2 overexpression compared with untransfected cells. In addition, the double transfection induced a more significant increase in cell proliferation compared with single transfections $(\mathrm{P}<0.01$ and $\mathrm{P}<0.001$; Fig. 3B and C). Furthermore, as presented in Fig. 3D, the results from Boyden chamber assay demonstrated that the migratory ability of EC109 cells was significantly increased following PRR11 or SKA2 overexpression compared with untransfected cells $(\mathrm{P}<0.01$; Fig. 3D). Cell migratory capacity was more significantly increased following overexpression of PRR11 and SKA2 compared with single transfections $(\mathrm{P}<0.001$; Fig. 3D). In addition, the results from transwell assay reported that overexpression of the gene pair significantly promoted the invasive ability of EC109 cells $(\mathrm{P}<0.01$ and $\mathrm{P}<0.001$; Fig. 3E).

PRR11 and SKA2 gene pair activates AKT and epithelialmesenchymal transition (EMT) signaling pathways. In order to explore the underlying mechanism of the gene pair PRR11 and SKA2 on ESCC progression, the protein expression of p-AKT, total AKT, Snial and N-cadherin of EMT, and Cyclin D1 of cell cycle signal in EC9706 and EC109 cells by western blotting. The results demonstrated that expression levels of p-AKT, Snail and N-cadherin were reduced following PRR11 and SKA2 knockdown in EC9706 cells, and double transfection induced a more notable decrease in the expressions of these proteins compared with single transfections (Fig. 4A). Opposite effects were observed in EC109 cells overexpressing PRR11 and SKA2 (Fig. 4B). In addition, PRR11 and SKA2 overexpression or knockdown had no effect on Cyclin D1 expression. Furthermore, PRR11 and 
A<smiles>C=C[C@H]1C=C[C@H]2C=C[C@H]12</smiles>

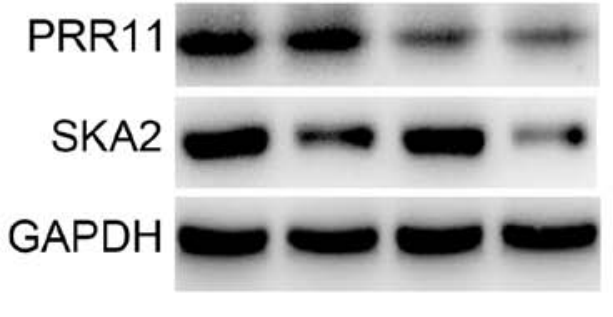

C

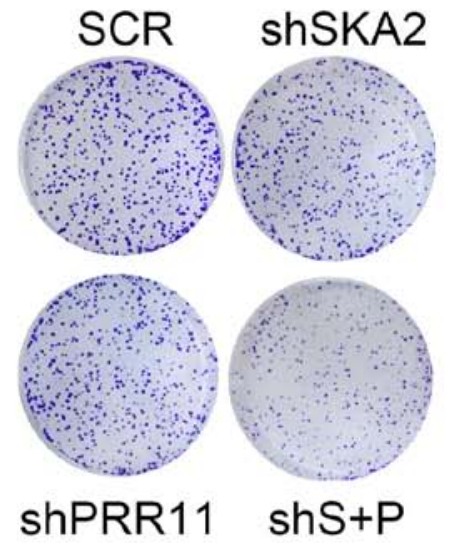

D
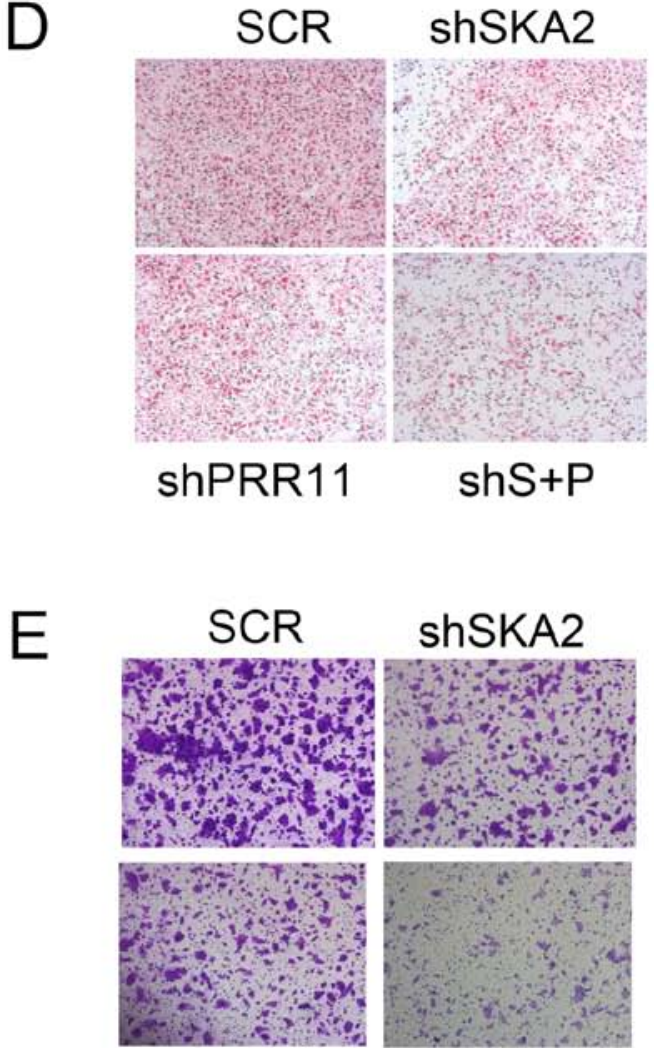

shPRR11

\section{shSKA2}
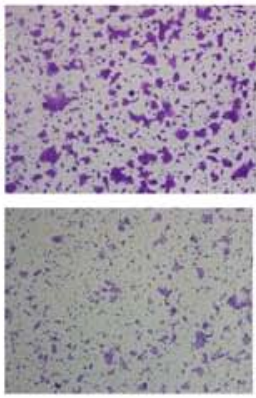

shS $+\mathrm{P}$
B
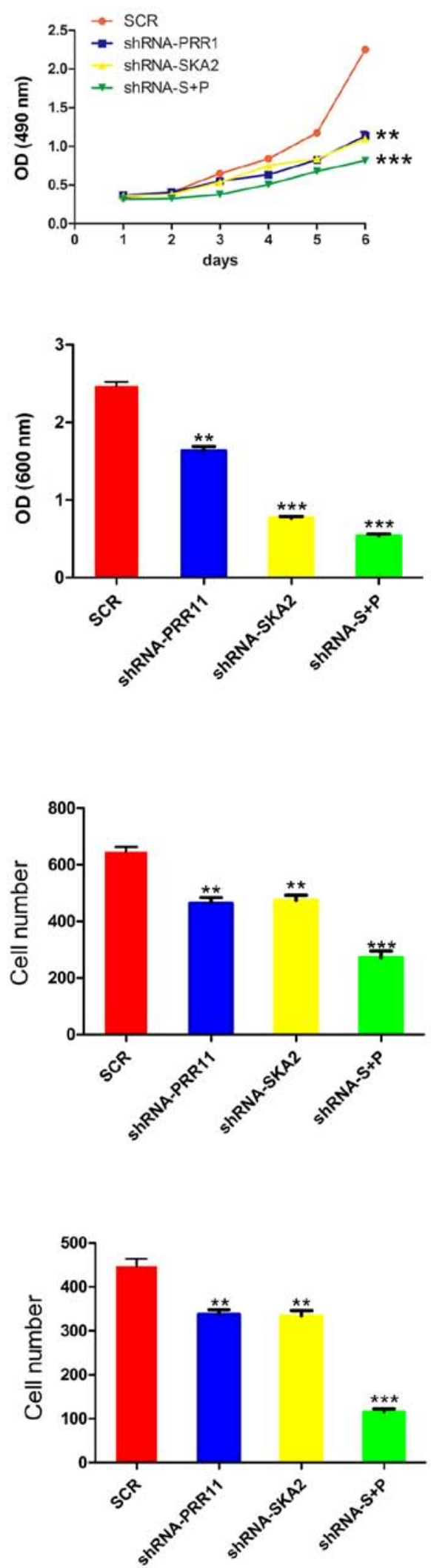

Figure 2. PRR11 or/and SKA2 knockdown inhibited proliferation, migratory and invasive capacities of ESCC cells. (A) Western blotting confirming PRR11 or/and SKA2 in EC9706 cells. (B) Effect of PRR11 or/and SKA2 knockdown on the proliferation of EC9706 cells assessed by MTT assay. (C) Effect of PRR11 or/and SKA2 knockdown on EC9706 cell proliferation assessed by crystal violet assay. (D) Effect of PRR11 or/and SKA2 knockdown on the migratory capacity of EC9706 cells assessed by Boyden chamber assay. (E) Effect of PRR11 or/and SKA2 knockdown on the invasive capacity of EC9706 cells assessed by transwell assay. Data were presented as mean \pm standard deviation. ${ }^{* *} \mathrm{P}<0.01$ and ${ }^{* * *} \mathrm{P}<0.001$. OD, optical density; P and PRR11, proline-rich protein 11 ; $\mathrm{S}$ and SKA2, spindle and kinetochore associated 2; SCR, scramble; Sh, short hairpin. 

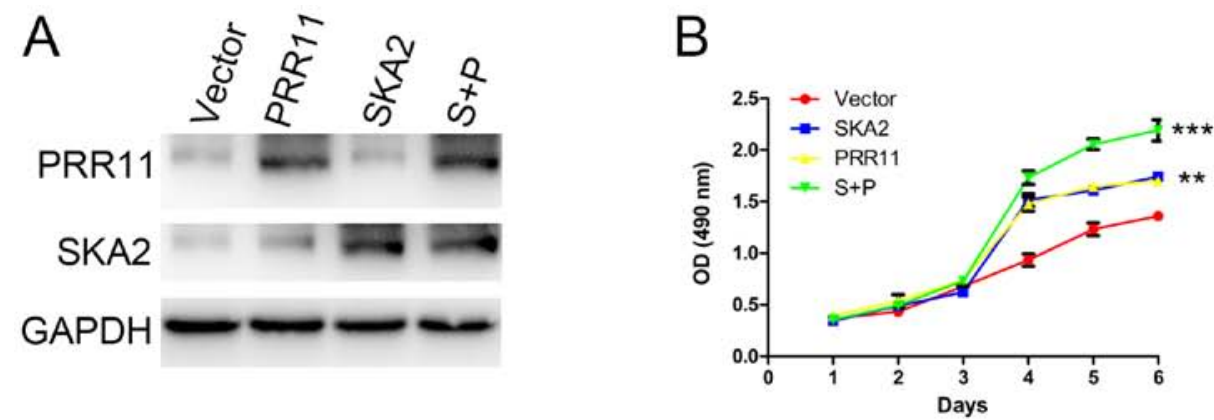

C
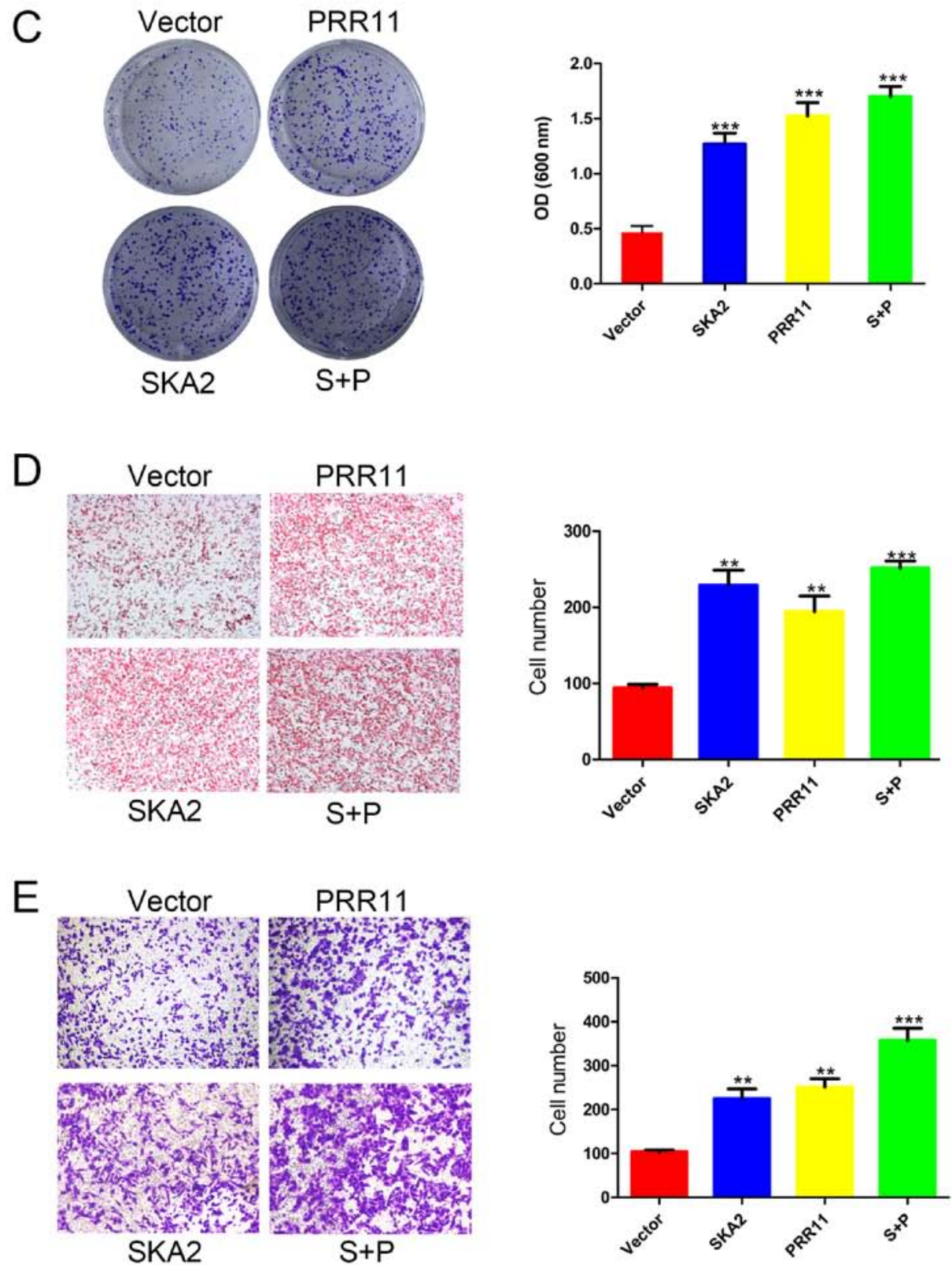

Figure 3. PRR11 or/and SKA2 overexpression promoted proliferation, migratory and invasive capacities of ESCC cells. (A) Western blotting confirming PRR11 or/and SKA2 overexpression in EC109 cells. (B) Effect of PRR11 or/and SKA2 overexpression on the proliferation of EC109 cells assessed by MTT assay. (C) Effect of PRR11 or/and SKA2 overexpression on EC9706 cell proliferation assessed by crystal violet assay. (D) Effect of PRR11 or/and SKA2 overexpression on the migratory capacity of EC109 cells assessed by Boyden chamber assay. (E) Effect of PRR11 or/and SKA2 overexpression on the invasive capacity of EC109 cells assessed by transwell assay. Data were presented as mean \pm standard error. ${ }^{*} \mathrm{P}<0.05,{ }^{* * *} \mathrm{P}<0.01$ and ${ }^{* * * *} \mathrm{P}<0.001$. OD, optical density; $\mathrm{P}$ and PRR11, proline-rich protein 11; S and SKA2, spindle and kinetochore associated 2; SCR, scramble; Sh, short hairpin.

SKA2 overexpression increased the expression of the proliferation marker PCNA, whereas their knockdown decreased PCNA expression (Fig. 4A and B).

\section{Discussion}

Over the past few decades, the prognosis of ESCC has been 
A

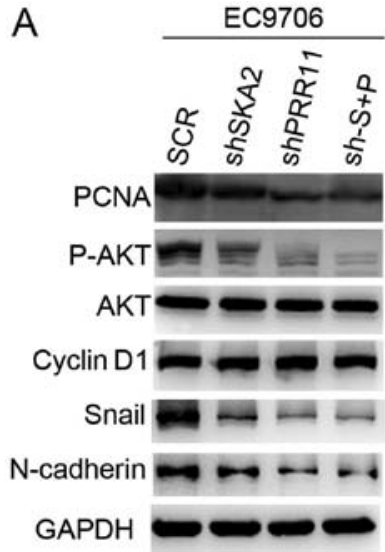

B

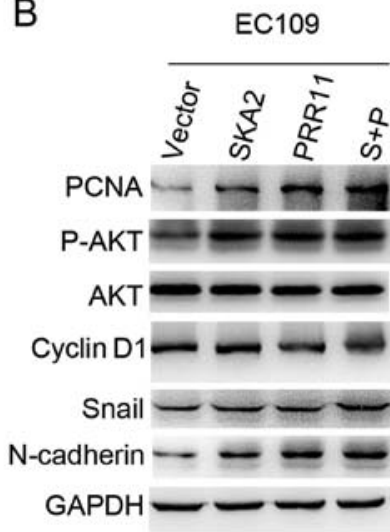

Figure 4. PRR11 or/and SKA2 overexpression promotes the AKT and EMT signaling pathways. (A) Effect of PRR11 or/and SKA2 knockdown on the expression of AKT, EMT-associated proteins and cell cycle-related proteins in EC9706 cells examined by western blotting. GAPDH was used as a loading control. (B) Effect of PRR11 or/and SKA2 overexpression on the expression of AKT, EMT-associated proteins and cell cycle-related proteins in EC109 cells examined by western blotting. GAPDH was used as a loading control EMT, epithelial-mesenchymal transition; PRR11, proline-rich protein 11; SKA2, spindle and kinetochore associated 2.

relatively poor in China, with the 5-year overall survival rate $<20 \%$ and most patients died within 1 year of diagnosis (2). In addition, this disease lacks sensitive and specific early diagnosis method. Surgical and radiation therapies are the main treatments for ESCC; however, numerous patients eventually develop advanced stages of ESCC (4). The present study aimed therefore to identify potential intervention targets for ESCC.

Recent studies reported that the gene pair of PRR11 and SKA2 is involved in tumorigenesis and cancer progression. Zhu et al (9) reported that PRR11 overexpression facilitates ovarian carcinoma cell proliferation, migration, and invasion through activation of the PI3K/AKT/ $\beta$-Catenin pathway. Furthermore, it was reported that the gene pair PRR11 and SKA2 is negatively regulated by $\mathrm{p} 53$ through nuclear factor $\mathrm{Y}$ in lung cancer cells (10). Also, PRR11 silencing leads to cell cycle arrest, suppresses colony formation, decreases cell proliferation and inhibits tumorigenic potential of lung cancer cells (19). In addition, the PRR11 and SKA2 gene pair has been hypothesized as a potential new target for the diagnosis and treatment of lung cancer (20). It has reported that overexpression of PRR11 could promote breast cancer progression by activating EMT (7). Qiao et al (6) demonstrated that proline-rich protein 11 silencing inhibits hepatocellular carcinoma growth and epithelial-mesenchymal transition through $\beta$-catenin signaling.

The present study demonstrated that PRR11 and SKA2 mRNA levels were significantly increased in ESCC tissues compared with adjacent normal tissues. Furthermore, cell proliferation, migratory and invasive capacities were significantly increased following PRR11 and SKA2 overexpression. In addition, PRR11 and SKA2 knockdown inhibited the proliferation, invasive and migratory capacities of ESCC cells. Subsequently, in order to investigate the underlying mechanism, this study demonstrated that PRR11 and SKA2 overexpression significantly activated the AKT signaling pathway and certain markers, including Snail and N-cadherin of EMT. AKT signaling pathway activation is implicated in the development of a numerous human cancers, including ESCC (21-23). Furthermore, EMT represents a critical event in the transition from early to invasive carcinomas, and it was demonstrated that $\mathrm{N}$-cadherin upregulation is associated with poor prognosis and lower survival in patients with cancer (24-26). These findings are consistent with the results from the present study. To the best of our knowledge, this study was the first to demonstrate the involvement of PRR11 and SKA2 in the progression of ESCC.

In summary, this study demonstrated that the gene pair PRR11 and SKA2 may serve a crucial role in the proliferation, migratory and invasive abilities of ESCC cells. These results provided a better understanding of the underlying mechanisms of PRR11 and SKA2 in ESCC tumor development, and PRR11 and SKA2 may be considered as potential targets for the diagnosis and/or treatment of ESCC.

\section{Acknowledgements}

Not applicable.

\section{Funding}

The present study was supported by the Young Core Instructor Foundation from the Education Commission of Henan Province (grant no. 2016GGJS-261) and the Science and Technology Project of Henan Province (grant no. 192102310103).

\section{Availability of data and materials}

The datasets used and/or analyzed during the current study are available from the corresponding author on reasonable request.

\section{Author's contributions}

JC and HY conducted the experiments. ZN conducted the experiments and analyzed the data. CK designed the research. $\mathrm{HZ}$ and RP analyzed the data. JC, RP and CK drafted the manuscript. All authors read and approved the final manuscript.

\section{Ethics approval and consent to participate}

The present study was approved by the Ethics Committee of the Second Affiliated Hospital of Zhengzhou University. All patients and provided signed informed consent.

\section{Patient consent for publication}

Not applicable.

\section{Competing interests}

The authors declare that they have no competing interests.

\section{References}

1. Bray F, Ferlay J, Soerjomataram I, Siegel RL, Torre LA and Jemal A: Global cancer statistics 2018: GLOBOCAN estimates of incidence and mortality worldwide for 36 cancers in 185 countries. CA Cancer J Clin 68: 394-424, 2018. 
2. Cheng YF, Chen HS, Wu SC, Chen HC, Hung WH, Lin CH and Wang BY: Esophageal squamous cell carcinoma and prognosis in Taiwan. Cancer Med 7: 4193-4201, 2018.

3. Ajani JA, D'Amico TA, Almhanna K, Bentrem DJ, Chao J, Das P, Denlinger CS, Fanta P, Farjah F, Fuchs CS, et al: Gastric cancer, version 3.2016, NCCN clinical practice guidelines in oncology. J Natl Compr Canc Netw 14: 1286-1312, 2016.

4. Chen J, Kwong DL, Cao T, Hu Q, Zhang L, Ming X, Chen J, $\mathrm{Fu} \mathrm{L}$ and Guan X: Esophageal squamous cell carcinoma (ESCC) Advance in genomics and molecular genetics. Dis Esophagus 28 : 84-89, 2015.

5. Walker RC and Underwood TJ: Molecular pathways in the development and treatment of oesophageal cancer. Best Pract Res Clin Gastroenterol 36-37: 9-15, 2018

6. Qiao W, Wang H, Zhang X and Luo K: Proline-rich protein 11 silencing inhibits hepatocellular carcinoma growth and epithelial-mesenchymal transition through $\beta$-catenin signaling. Gene 681: 7-14, 2019.

7. Wang Y, Zhang C, Mai L, Niu Y, Wang Y and Bu Y: PRR11 and SKA2 gene pair is overexpressed and regulated by p53 in breast cancer. BMB Rep 52: 157-162, 2019

8. Zhang L, Lei Y, Zhang Y, Li Y, Bu Y, Song F and Zhang C: Silencing of PRR11 suppresses cell proliferation and induces autophagy in NSCLC cells. Genes Dis 5: 158-166, 2017.

9. Zhu J, Hu H, Wang J, Yang Y and Yi P: PRR11 overexpression facilitates ovarian carcinoma cell proliferation, migration, and invasion through activation of the PI3K/AKT/ $\beta$-catenin pathway. Cell Physiol Biochem 49: 696-705, 2018.

10. Wang Y, Zhang Y, Zhang C, Weng H, Li Y, Cai W, Xie M, Long Y, Ai Q, Liu Z, et al: The gene pair PRR11 and SKA2 shares a NF-Y-regulated bidirectional promoter and contributes to lung cancer development. Biochim Biophys Acta 1849: $1133-1144,2015$.

11. Chan YW, Jeyaprakash AA, Nigg EA and Santamaria A: Aurora B controls kinetochore-microtubule attachments by inhibiting Ska complex-KMN network interaction. J Cell Biol 196: 563-571, 2012.

12. Zhang Q, Sivakumar S, Chen Y, Gao H, Yang L, Yuan Z, Yu H and Liu H: Ska3 phosphorylated by Cdk1 binds Ndc80 and recruits Ska to kinetochores to promote mitotic progression. Curr Biol 27: 1477-1484.e4, 2017.

13. Jeyaprakash AA, Conti E, Santamaria A, Jayachandran U, Chan YW, Benda C and Nigg EA: Structural and functional organization of the Ska complex, a key component of the kinetochore-microtubule interface. Mol Cell 46: 274-286, 2012.

14. Hanisch A, Silljé HH and Nigg EA: Timely anaphase onset requires a novel spindle and kinetochore complex comprising Ska1 and Ska2. EMBO J 25: 5504-5515, 2006.

15. Vaidya T, Desai S and Mahajan A: 8th edition AJCC and imaging TNM: Time to break-in and assert in the staging process! Indian J Cancer 56: 271-273, 2019.
16. Livak KJ and Schmittgen TD: Analysis of relative gene expression data using real-time quantitative PCR and the 2(-Delta Delta C(T)) method. Methods 25: 402-408, 2001.

17. Yang J, Antin P, Berx G, Blanpain C, Brabletz T, Bronner M, Campbell K, Cano A, Casanova J, Christofori G, et al: Guidelines and definitions for research on epithelial-mesenchymal transition. Nat Rev Mol Cell Biol, Apr 16, 2020 (Epub ahead of print).

18. Leal-Esteban LC and Fajas L: Cell cycle regulators in cancer cell metabolism. Biochim Biophys Acta Mol Basis Dis 1866: 165715 , 2020.

19. Ji Y, Xie M, Lan H, Zhang Y, Long Y, Weng H, Li D, Cai W, Zhu H, Niu Y, et al: PRR11 is a novel gene implicated in cell cycle progression and lung cancer. Int J Biochem Cell Biol 45: 645-656, 2013.

20. Zhang C, Zhang Y, Li Y, Zhu H, Wang Y, Cai W, Zhu J, Ozaki T and $\mathrm{Bu}$ Y: PRR11 regulates late-S to $\mathrm{G} 2 / \mathrm{M}$ phase progression and induces premature chromatin condensation (PCC). Biochem Biophys Res Commun 458: 501-508, 2015.

21. Liu X, Song M, Wang P, Zhao R, Chen H, Zhang M, Shi Y, Liu K, Liu F, Yang R, et al: Targeted therapy of the AKT kinase inhibits esophageal squamous cell carcinoma growth in vitro and in vivo. Int J Cancer 145: 1007-1019, 2019.

22. Li J, Chen Y, Zhan C, Zhu J, Weng S, Dong L, Liu T and Shen X: Glypican-1 promotes tumorigenesis by regulating the PTEN/Akt/ $\beta$-catenin signaling pathway in esophageal squamous cell carcinoma. Dig Dis Sci 64: 1493-1502, 2019.

23. Zhang J, Fa X and Zhang Q: MicroRNA-206 exerts anti-oncogenic functions in esophageal squamous cell carcinoma by suppressing the c-Met/AKT/mTOR pathway. Mol Med Rep 19: 1491-1500, 2019.

24. Rosanò L, Spinella F, Di Castro V, Nicotra MR, Dedhar S, de Herreros AG, Natali PG and Bagnato A: Endothelin-1 promotes epithelial-to-mesenchymal transition in human ovarian cancer cells. Cancer Res 65: 11649-11657, 2005.

25. Cano A, Pérez-Moreno MA, Rodrigo I, Locascio A, Blanco MJ, del Barrio MG, Portillo F and Nieto MA: The transcription factor snail controls epithelial-mesenchymal transitions by repressing E-cadherin expression. Nat Cell Biol 2: 76-83, 2000.

26. Barberà MJ, Puig I, Dominguez D, Julien-Grille S, GuaitaEsteruelas S, Peiro S, Baulida J, Francí C, Dedhar S, Larue L and García de Herreros A: Regulation of Snail transcription during epithelial to mesenchymal transition of tumor cells. Oncogene 23: 7345-7354, 2004.

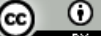

This work is licensed under a Creative Commons Attribution 4.0 International (CC BY 4.0) License. 